\title{
Estrategias didácticas de la Educación Física para desarrollar la motivación en estudiantes de Educación Escolar
}

\section{Didactic strategies of Physical Education to develop motivation in School Education students}

\author{
Julio Luis Peñafiel Álvarez ${ }^{1}$, Sergio Constantino Ochoa Encalada ${ }^{1}$ y Diego Andrés Heredia León ${ }^{1}$ \\ ${ }^{1}$ Universidad Católica de Cuenca, Ecuador \\ *jlpenafiela@ucacue.edu.ec
}

DOI: https://doi.org/10.26871/killkanasocial.v4i2.615

\begin{abstract}
Resumen
La presente investigación tiene como objetivo proporcionar las técnicas, las estrategias, los estilos de enseñanza, y la motivación que el docente de Educación Física debe conocer y poner en práctica en sus actividades diarias al momento de impartir las clases. La finalidad es que con estas herramientas sus discentes reciban clases dinámicas, activas y de esta forma contribuir al desarrollo de la formación integral, en los ámbitos cognitivo, afectivo, social y emocional, y adquirir ciertos niveles de autonomía que les permitirá tomar decisiones dentro del proceso de inter-aprendizaje y la práctica social. El diseño es de tipo bibliográfico. Se conoce que los niños y jóvenes realizan actividad física en muchos casos porque el maestro obliga a la ejecución de un solo tipo de ejercicio o algún juego que no despierta mayor interés, sin divertirse ni relajarse y peor aún sentirse parte del grupo o preocuparse por su salud. Se debe tener en cuenta que la motivación es fundamental dentro de los espacios académicos, la utilización de estrategias favorables pueden generar cambios en la conducta del estudiantado, de lo contrario el tratamiento de esta disciplina deportiva será solo por cumplir con el pensum y el horario establecido, como se da en la mayoría de centros educativos, que en muchos casos no trabaja un profesional de la rama sino docentes que no tienen la formación científica y que únicamente improvisan, lo que genera en el estudiantado la apatía y el desinterés por la asignatura.
\end{abstract}

Palabras clave: Deporte escolar, estrategias de enseñanza, motivación, disciplina deportiva, actividad física.

\begin{abstract}
The purpose of this research is to provide Physical Education teachers with the techniques, strategies, teaching styles, motivation that the teacher must know and put into practice in their daily activities when teaching, in order for their students to leave. of the routine, do not fall into boredom and contribution to the development of integral training, in the cognitive, affective, social and emotional parameters of autonomy that allows them to make decisions within the learning process and social practice. The design is of bibliographic type, it is known that children and young people perform physical activity in many cases by the mandatory teacher to the execution of a single type of exercise or some game that does not arouse greater interest, without having fun or relaxing and worse still feeling part of the group or worry about their health, should take into account that motivation is essential within academic spaces, the use of favorable strategies so that they can generate changes in the conduct of the study, otherwise the treatment of this discipline Sports will be determined by complying with the thought and the established schedule, as it has been taking place in most of the educational centers, which in many cases does not work a professional of the branch but teachers who do not have scientific training and who are totally improvising, generating in the student apathy and disinterest in the subject.
\end{abstract}

Keywords: School sports, teaching strategies, motivation, sports discipline, physical activity.

\section{Introducción}

El presente trabajo tiene por objeto poner a consideración de los docentes de Educación Física, técnicas, métodos y estilos de enseñanza que se pueden poner en práctica en clases de esta área. El uso de estrategias de enseñanza en la asignatura de Cultura Física especialmente en los niños, contribuyen al desarrollo de destrezas, ha- bilidades y la motivación por la actividad deportiva; es conveniente manifestar que existe un para qué y porqué de la enseñanza y en eso, debe existir la aplicación de las estrategias pedagógicas por parte del docente en la clase; y, de esta forma tiende a ser activa y motivacional. Para que el estudiante tenga una empatía con la actividad física; desde esta perspectiva se atiende a los intereses, necesidades y 
sobre todo se potencializaría las habilidades y capacidades de los alumnos (Fernández, 2004)

Varios docentes pretenden hacer bien las cosas dentro del proceso de enseñanza de educación física; por lo que el profesor debe tener conocimiento en primer lugar sobre el tema que va a impartir al estudiante, a partir de ello debe ser capaz de aplicar las estrategias o métodos necesarios para que se produzca el proceso enseñanza-aprendizaje obtenido los resultados esperados Escolano (2012) que permite descubrir diferentes técnicas, tácticas y modos de trabajar dando lugar a la utilización de varios estilos de enseñanza.

Se entiende como estilos de enseñanza desde aportes de Alarcon, T. y Reyno, M. (2012) "al conjunto de interacciones didácticas de tipo técnico, organizacional-control y socio-afectivo desarrollados durante la clase" (p.15); fundamentando en lo expuesto y lo que expresa las normativas al respecto; la educación del siglo XXI necesita la utilización de técnicas activas con el fin de potencializar las distintas destrezas adquiridas, y para esto el estudiante debe estar motivado hacia este aprendizaje de calidad.

Con base en la investigación bibliográfica que se realiza se conoce de artículos con esta temática escritos en el España, entre ellos tenemos "Motivación en educación física, a través de diferentes metodologías didácticas (2017). "Estrategias pedagógicas para la potenciación de la iniciación deportiva en la educación física (2017) "Fomento de la Actividad Física en México" (2015). En el Ecuador no se han identificado artículos que aborden este tema; de allí la importancia, de analizar las estrategias didácticas motivacionales de la actividad física para escolares desde este contexto.

De acuerdo con la Ley del Deporte en el Art. 81 indica que "La Educación Física... Busca formar de una manera integral y armónica al ser humano, estimulando positivamente sus capacidades físicas, psicológicas, éticas e intelectuales, con la finalidad de conseguir una mejor calidad de vida. (Ministerio del deporte, 2010); esta asignatura tiene la misión en los distintos establecimientos de nuestro País contribuir al desarrollo de la personalidad de niños (as), adolescentes y jóvenes; exclusivamente preparar para un buen vivir; ante esto Arjona, Checa y Pardo dice al respecto "el deporte puede ser un medio para trasmitir valores personales y sociales como respeto, autoestima, empatía, esfuerzo, autonomía, cooperación, ayuda a los demás" (Arjona Garrido, Checa Olmos, y Pardo García, 2016)

La Ley de Deporte, Educación Física y Recreación en su Art. 11 menciona que "La práctica del deporte, educación física y recreación. - Es derecho de las y los ciudadanos practicar deporte, realizar educación física y acceder a la recreación, sin discrimen alguno" (Ministerio del deporte, 2010, p. 4), (Ainscow, 2001) de acuerdo a la Constitución de la República y a la presente Ley, el objetivo principal de esta normativa es brindar la garantía y la práctica adecuada y amparada por el Estado, considerándose como un derecho inalienable de las personas para conseguir ese desarrollo integral tan anhelado por el pueblo ecuatoriano.

Es preocupación de la población ecuatoriana, y en especial de la provincia del Cañar; que en la mayoría de los establecimientos educativos no están impartiendo esta asignatura, docentes especializados en la rama es así que el $90 \%$ de profesionales no son titulados para desempeñar estas funciones de acuerdo al Plan de Desarrollo Provincial; al no ser especialistas en su cátedra, la mayoría de profesores no conocen de la estrategias didácticas que deben poner en práctica en la clase y por ende no hay interés de los estudiantes en esta importante asignatura que es fundamental para la formación integral de la personalidad.

En la LOEI y su reglamento, la carga horaria estipula en 5 horas pedagógicas semanales en todos los tres niveles (elemental, media y superior), pero que no solo quede en el campo recreativo o por cubrir horas semanales, sino que al contrario se imparta con estrategias apropiadas motivadoras para el estudiantado; en la mayoría de establecimientos educativos los que imparten estas horas son los mismos docentes que están a cargo del año de básica; dichos docentes son profesionales de Educación Básica, pero no tienen conocimiento de Educación Física (Distrito 03D01: Azogues-Biblián-Déleg)

Aportar con estrategias de como impartir clases en Educación Física a personas que desconocen esta área es de vital importancia, conviene recalcar que no existe muchas investigaciones nacionales ni locales al respecto, por lo que es impostergable el tratamiento estratégico de esta disciplina.

En la adolescencia y juventud muchas personas participan o se interesan en desarrollar ciertas actividades deportivas; al respecto, nos preguntamos ¿por qué ese interés? y ¿cuál es la razón de su apatía?; existen diversos motivos de porque los inicios y abandonos de la práctica del deporte o la realización de actividad física; unos puede ser por mejorar su condición de vida, de afiliación, de diversión y otros debido a que se desarrolla el aspecto físico y psicológico del ser humano y sobre todo reduce un buen número de problemas de salud y sicosociales que a la larga ocasiona grandes problemas para el estado.

\section{Metodología}

El diseño es de tipo bibliográfico, se realizó la búsqueda de información y revisión de fuentes en varios artículos científicos, no se aplicó ningún instrumento de recolección de datos.

\subsection{Estrategias de Enseñanza en Educación Física}

La estrategia siempre está presente en toda actividad humana, por lo que se considera como una planificación para solucionar cualquier problema que se presente en nuestra vida cotidiana. Se puede considerar que estrategia es producto de un acto creativo, innovador, lógico y aplicable para un determinado plan, proceso, proyecto o una acción que va a ejecutar en cualquier circunstancia que se 
presente; para lo cual genera una serie de objetivos, tácticas y por ende recursos que son destinados a solucionar el conflicto; la elaboración de estrategias es un conjunto de fines, metas, objetivos, recursos y evaluaciones que pone en práctica las personas o instituciones que quieren solucionar un problema o necesidad presentada en su ámbito respectivo de trabajo. Ante esto Valle, define: Estrategia: “... acciones secuenciales e interrelacionadas que partiendo de estado inicial dado el diagnóstico permite dirigir el paso a un estado ideal consecuencia de la planeación" (Valle, 2012) por lo que se puede decir que es un plan ideado por los entrenados para coordinar las acciones deportivas y maniobras necesarias para lograr un fin. De acuerdo a esta descripción las estrategias no pueden aparecer de la nada, sino después de haber aplicado una serie de métodos y técnicas escritas al respecto; después de este proceso el docente (en este caso) está en la capacidad de adecuar, crear o mejorar ciertas actividades de acuerdo a su contexto y poner en práctica en orden lógico y sistemático y en esta forma conseguir su objetivo o su meta planteada.

La estrategia pertenece al orden de la concepción (crea el entrenador), mientras que la táctica corresponde al de la ejecución (participación de los deportistas). El concepto de estrategia deportiva se basa en un planteamiento priori, anticipando situaciones, que se dan o se podrían dar en las competiciones consiguiendo así la motivación para un verdadero disfrute, integrándole en un estilo de vida saludable frente al sedentarismo González (2017) Al aplicar estos conceptos, los alumnos experimentarán e irán adquiriendo competencias y autonomía que les permitirá tomar decisiones dentro del proceso de inter-aprendizaje y de práctica social.

\subsection{Estrategias de Competencia}

Tiempo. - Con el fin que el tiempo sea adecuado para desarrollar la destreza con criterio de desempeño en la educación escolar, especialmente educación básica; el docente de Educación Física debe realizar las planificaciones tomando en consideración estos aspectos; los tres niveles de la concreción del Currículo (macro, mezo y micro); dando mayor importancia al último nivel, especialmente a la Unidad Didáctica y el Plan de clases; considerar la Planificación Curricular Anual, especialmente sus objetivos de año y desde aquí la unidad didáctica y el plan diario y los períodos de clases que va a utilizar en esa unidades; el docente tiene 40 semanas laborables descontando el $10 \%$ para imprevistos y evaluación en un año lectivo, en donde sabemos cuántas horas semanales tenemos que trabajar en esta asignatura y esto debe tener íntima relación con las destrezas a desarrollar por lo que tenemos que dividir este tiempo de acuerdo al grado de dificultad cuántos períodos de clases se asigna a cada una de estas destrezas y con qué estrategias tengo que trabajar. Hay que recalcar uno de los aspectos muy importante de la planificación curricular es la evaluación; la mayoría de docentes no realizan esta planificación, las mismas que deben ser planificados sus criterios al inicio del año lectivo de acuerdo a lo que exige el Reglamento a la LOEI en su artículo 205. Asamblea Nacional (2015) porque una buena práctica de la actividad física debe realizarse bajo ciertos parámetros así obtendrá efectos saludables, caso contrario no tendría ningún valor sino se considera este factor muy importante.

Actividades Novedosas. - El docente siempre debe planificar actividades innovadoras y creativas para desarrollar ciertas capacidades que tienen los estudiantes dentro de la actividad deportiva; nunca el profesor debe caer en la rutina, el aburrimiento, el ocio; originando poco a poca desinterés hacia las actividades que se están realizando porque esa apatía, desmotivación, desgano; anula la participación, considerando que no aporta mayormente en su vida; nunca como profesores debemos permanecer en la zona de confort, siempre se debe estar en la zona de riesgo porque es ahí donde se brinda un aprendizaje eficiente, eficaz y efectivo; existe muchas actividades que se puede ejecutar para desarrollar ciertas competencias. Realizar cualquier práctica deportiva no tiene porqué ser obligado y aburrido, en la actualidad todas estas actividades deben ser divertidas y sanas; las sesiones de entrenamiento deben ser adaptadas a los gustos y sobre todo a las necesidades físicas de cada uno, llegando al disfrute mientras realiza la actividad física.

Establecer Reglas. - Todo docente al inicio de cada período o ciclo debe establecer reglas en donde consten acuerdos y compromisos que tienen que estar en íntima relación con los objetivos de año y de las unidades didácticas; en Educación Física es conveniente que para cada actividad debe establecerse reglas con el fin de que la evaluación sea objetiva, utilizando instrumentos de evaluación pertinentes para el caso; en esta forma todos los estudiantes estaría conscientes de sus respectivos avances acorde a sus capacidades físicas e intelectuales, es fundamental que de estas reglas los alumnos se concienticen de que en la vida todos debemos desempeñar ciertos roles en diferentes momentos de la existencia y las reglas constituye un recordatorio para tener presente que debemos vivir y disfrutar respetando todo lo que nos rodea.

Valorar el esfuerzo y la superación personal. - Es necesario que el docente, de mayor importancia a la dedicación, empeño, esfuerzo y sobre todo a la responsabilidad de cumplir con su rol y tratar de ser competente en su función; porque se observa con frecuencia un alto rendimiento en el entrenamiento pero baja en el momento de la competencia cometiendo errores garrafales Llames R. García A. (2017) es recomendable recordar que vivimos en una sociedad capitalista en donde la competencia en la sociedad es diaria, por lo que desde el ambiente de clases es necesaria preparar para este tipo de vida que llevamos, en donde debemos aprender a disfrutar nuestros logros y corregir errores a través de la retroalimentación y el refuerzo de ciertas actividades o destrezas a desarrollar. A pesar que la sociedad actual en ciertos casos apunta a la comodidad sin permitir que la persona salga de la zona de confort, llevando a la niñez y juventud a conseguir lo que persiguen sin mayor 
esfuerzo, lo que es urgente que el docente despierte en sus estudiantes afrontar las dificultades y enseñarles el valor del esfuerzo a partir de una fuerza de voluntad.

Reconocer el progreso individual. - Es saludable que el docente informe al estudiante como va avanzando con evidencias respectivas, esto es conveniente con el fin que siga esforzándose y llegue a lo óptimo dentro de la preparación física, partiendo que cada individuo desarrolla sus potencialidades de forma diferencial, implementando actividades que desarrollen las habilidades personales.

Mejora permanente del estudiante. - Dweck (2013) describe dos creencias en cuanto a la habilidad. Creencia de entidad, que sus habilidades son naturales y que por mucho que se esfuerce no van a conseguir mejoras. Creencia incremental, que si se esfuerza y trabaja duro seguro que va hacer cada vez mejor un deportista, asimismo manifiesta que de acuerdo a estas creencias es la actuación del dicente, hay muchos de ellos que manifiestan profesor no podemos, como se hace específicamente, no me sale, realmente no puedo, etc. Expresiones como estas son a diario cuando tienen la primera creencia; entonces hay que incentivar para ir por la segunda cuidando la integridad de cada uno de ellos y sobre todo siguiendo un proceso adecuado que no de origen a lesiones en ningún estudiante.

Evitar agrupar al alumnado siguiendo criterios de competencia. - Es didáctico que el docente en la mayoría de casos forme los grupos dentro de una clase, estará conformado por estudiantes que desempeñen diferentes roles con el fin que se dé equilibrio en el ambiente de clases, caso contrario existirá los grupos que sobresalen y otros que quedarán en la mediocridad o son pésimos; es conveniente analizar capacidades de cada uno y mezclarlo con el fin que se de esta sana competencia.

\subsection{Estrategias para fomentar la autonomía}

Dar la posibilidad de elección y fomentar la participación en el proceso de toma de decisiones. - El docente de educación física debe facilitar opciones al estudiante a que escoja dentro del proceso de formación, en donde prime la responsabilidad entre el estudiantado, esta no sólo es una constante en los estatutos y leyes que rigen a la nación como Ley Orgánica de Educación y Diseño Curricular Base, sino que es una responsabilidad grande de todos quienes están inmersos en proceso de inter- aprendizaje para el desarrollo de la autonomía Martos, et al. (2014)

Objetivos. - Debe proponer el docente que cada estudiante sugiera su objetivo en esta forma fomenta la percepción de competencia y su autonomía.

Contenidos. - Considerar un conjunto de contenidos relacionados con el objetivo planteado y el alumno escoja de acuerdo con sus propias reglas de juego su gusto y sobre todo que ayude a cumplir con lo planteado, es necesario seleccionar las actividades de acuerdo con sus necesidades y capacidades.
Reglas de juego. - Los estudiantes pueden crear sus propias reglas y normas de juegos, pero sin salir del objetivo específico planteado al inicio de la actividad.

Espacio. - Si disponemos de diferentes instalaciones podemos dar a elegir donde trabajar.

Tiempo. - En las diferentes clases es conveniente dar a elegir el tiempo para que se planteen las actividades respectivas, pero para esto debe ser consciente el estudiante que el objetivo tiene que cumplirse.

Técnicas de enseñanza y estrategia en la práctica. - Es conveniente que el docente utilice diferentes técnicas y estrategias de aprendizaje y desde esta perspectiva dar a elegir al estudiante que técnica o táctica que quieren desarrollar cierta habilidad o destreza necesaria para cumplir con el objetivo de esa clase; es conveniente señalar que la acción o actividad a realizarse tiene íntima relación con el método o técnica a desarrollarse.

Evaluación. - El estudiante debe participar en diseño de la evaluación tomando en cuenta los indicadores planteados al inicio del ciclo o año.

Explicar los objetivos didácticos de cada actividad. - En cada inicio de un ciclo, unidad o clase es necesario manifestar que es lo que perseguimos en esa sesión, esto hace que el dicente sea menos controlado y más autónomo.

Recompensas limitadas. - De acuerdo a la teoría clásica de Watson (estímulo respuesta), la mayoría de acciones y estrategias se planifica en función a la respuesta (positiva); de este modo el reforzamiento (regalo o prebenda) esta debe ser limitado; de acuerdo a Jean Piaget es aconsejable hasta los cinco años y desde esta edad ir disminuyendo con el fin que el estudiante haga conciencia que toda acción que está cumpliendo dentro de una estrategia, posteriormente va ir en beneficio del que practica y en esta forma estaríamos guiando al alumno hacia la práctica autónoma.

Utilizar estilos de enseñanza participativo. - De acuerdo a la nueva teoría (constructivista) la estrategia que mejor se adapta a esta sociedad son las técnicas activas y sobre todo desarrollan la autonomía; entre las técnicas que se practican en Educación Física encontramos; enseñanza recíproca, trabajos por grupos reducidos, estilo de micro enseñanza, etc.

Estilos de Enseñanza de la Indagación. - Esta técnica es parte del estilo cognitivo; su proceso es realizar un conjunto de preguntas por parte del guía en forma lógica y procesal dentro de la clase, su objetivo es para verificar si han comprendido la o las actividades que tienen que ejecutar; y sobre todo lleva al estudiante a descubrir la respuesta adecuada.

La utilización de los estilos de enseñanza depende del contenido que se imparte, de las capacidades, intereses y necesidades de los estudiantes y en función de este se utilizará el estilo adecuado o la solución de un problema planteado.

\subsection{Estrategias para fomentar la relación}

a) Relación alumno-alumno 
Desarrollar la integración grupal. - La asignatura de Educación Física permite fomentar las relaciones sociales entre los alumnos, se debe plantear tareas cooperativas con un objetivo común en donde todos los integrantes del grupo tengan que desempeñar sus roles para poder conseguirlo; dentro de estas técnicas tenemos trabajos por pares recíprocos. No se debe olvidar la responsabilidad del docente, de eliminar los valores negativos tales como la rivalidad, el egocentrismo y el individualismo González (2017)

Agrupación flexibles y heterogéneas. - El docente para el trabajo de grupos debe utilizar diferentes tácticas entre estas puede formar los grupos al azar, equilibrados, partiendo de sus capacidades, en forma libre, etc., no es necesario abusar ninguno de ellos, sino que tiene que ser rotativo.

Evitar la rivalidad entre compañeros y desarrollar el autocontrol. - Es necesario educar en el autocontrol para que no hagan algo que tengan que arrepentirse o generar problemas.

b) Relación profesor-alumno

Crear un clima de preocupación y ser inteligente emocionalmente. - el concepto de clima de preocupación hace que se interese por el estudiante, inclusive fuera del aula; uno de los objetivos de esta técnica es que el docente se preocupe por sus estudiantes y no únicamente en la institución educativa, sino fuera de ella, esto haría que medite, reflexione y en esta forma pensará para ejecutar tal o cual acción convirtiéndose en un influyente en la vida de las personas que brinde confianza y apoyo a todo el estudiantado; el fin esencial constituye identificar las causas disruptivas, considerando las circunstancias que rodean al alumnado GanoOverway, L., y Méndez Giménez, A. (2014).

Mostrar pasión. - Se conoce que el maestro que trabaja con entusiasmo, establece empatía y la actividad que realiza, los estudiantes asimilan con gusto y motivación. La pasión se asocia con las emociones ocasionando un resultado positivo y por ende mejora las relaciones personales y mayor satisfacción en la vida Vallerand, R. J. Roberts G.C. (2015) El docente que hace de su profesión una forma de vida, es seguro que lo que haga en clase será con empeño, responsabilidad y sobre todo con ímpetu, en esta forma disfrutará de lo que ejecuta y siempre estará dispuesto a la actualización y el cambio.

Tener en cuenta la importancia motivacional de cada sesión. - La motivación situacional influye de manera significativa en la motivación contextual, el profesor de educación física valora cada uno de los momentos en los que interactúa con el educando, ya que todos esos momentos son relevantes motivacionalmente.
Tabla 1: Definición de términos didácticos

\begin{tabular}{ll}
\hline \multicolumn{1}{c}{ Términos Didácticos } & \multicolumn{1}{c}{ Conceptos } \\
\hline Didáctica & $\begin{array}{l}\text { Disciplina que ayuda y guía en el proceso de enseñanza- } \\
\text { aprendizaje }\end{array}$ \\
Modelo & $\begin{array}{l}\text { Término teórico que ayuda a entender mejor elementos y } \\
\text { situaciones del aprendizaje }\end{array}$ \\
Estilo de Enseñanza & $\begin{array}{l}\text { Término más correcto que el de Estrategia Pedagógica o } \\
\text { Estrategia Didáctica, haciendo referencia a cómo el } \\
\text { profesor presenta la materia }\end{array}$ \\
Método & $\begin{array}{l}\text { Término ambiguo utilizado principalmente para hacer } \\
\text { referencia a la forma de llevar a cabo la enseñanza }\end{array}$ \\
Intervención Didáctica & $\begin{array}{l}\text { Acciones del profesor a la hora de llevar a cabo el proceso } \\
\text { de enseñanza-aprendizaje }\end{array}$ \\
Técnicas de enseñanza & $\begin{array}{l}\text { Todo lo relacionado con la interacción y comunicación } \\
\text { profesor-alumno }\end{array}$ \\
\hline
\end{tabular}

Fuente: Adaptado de (Fernández-Rivas y Espada-Mateos, 2016)

Tabla 2: Estrategia o estilo de enseñanza

\begin{tabular}{|c|c|c|}
\hline $\begin{array}{c}\text { Estilo de } \\
\text { Enseñanza o } \\
\text { Estrategia } \\
\end{array}$ & Técnica & Concepto \\
\hline \multirow{4}{*}{$\begin{array}{l}\text { Tradicional } \\
\text { (ligados a una } \\
\text { visión } \\
\text { mecanicista del } \\
\text { cuerpo) }\end{array}$} & Mando Directo & $\begin{array}{l}\text { "El profesor está continuamente ejecutando porque es el referente de la } \\
\text { tarea en cada momento, solamente predomina la información inicial de } \\
\text { la tarea, no hay feedback individual. }\end{array}$ \\
\hline & $\begin{array}{l}\text { Modificación del } \\
\text { Mando Directo }\end{array}$ & $\begin{array}{l}\text { No hace falta que el profesor indique verbalmente el inicio del ejercicio } \\
\text { ya que el alumno está continuamente observando al profesor, por lo } \\
\text { tanto, sólo tiene que seguir e imitar. No todos al mismo ritmo } \\
\text { necesariamente. }\end{array}$ \\
\hline & Asignación de & $\begin{array}{l}\text { Sustituimos el modelo de ejecución del profesor por una información } \\
\text { inicial verbal y visual sobre la tarea a realizar. Durante la ejecución no } \\
\text { tiene presente el modelo. }\end{array}$ \\
\hline & & $\begin{array}{l}\text { No existe individualización, aunque si una adaptación cuidadosa al nivel } \\
\text { medio del grupo (enseñanza masiva). }\end{array}$ \\
\hline \multirow{3}{*}{$\begin{array}{l}\text { Enseñanza por } \\
\text { Posibilidad de } \\
\text { Participación }\end{array}$} & $\begin{array}{l}\text { Enseñanza } \\
\text { Recíproca }\end{array}$ & $\begin{array}{l}\text { Se basa en trabajo por parejas a lo largo de toda la Unidad se mantiene } \\
\text { eso, pero las parejas si se pueden cambiar. Donde hay dos roles: } \\
\text { ejecutante y observador y se va rotando. }\end{array}$ \\
\hline & $\begin{array}{c}\text { Grupos } \\
\text { Reducidos }\end{array}$ & $\begin{array}{l}\text { No se pude confundir con enseñanzas por grupos de nivel. Aquí los } \\
\text { grupos los establezco por roles: Rol de ejecutante. Rol de observador. } \\
\text { Rol de ayuda física (en gimnasia deportiva). Rol de evaluador (me } \\
\text { limito a ponderar el rol de ejecución). }\end{array}$ \\
\hline & Micro Enseñanza & $\begin{array}{l}\text { Implica una iniciación a la función docente porque la estructura sería la } \\
\text { siguiente: } 25 \text { alumnos; se forman } 4 \text { grupos con } 5 \text { alumnos y cada grupo } \\
\text { es enseñado a su vez por otro alumno. Aquín no se rota. Implica que el } \\
\text { profesor cede al alumnono líder la responsabilidad completa de la } \\
\text { enseñanza de los compañeros. }\end{array}$ \\
\hline \multirow{4}{*}{$\begin{array}{l}\text { Enseñanza que } \\
\text { Fomenta la } \\
\text { Individualización }\end{array}$} & $\begin{array}{l}\text { Individualización } \\
\text { por grupos }\end{array}$ & $\begin{array}{l}\text { Necesidad de distintos niveles, distintos intereses o ambos en el mismo } \\
\text { grupo; en función de esto se establecen dos o más grupos de trabajo } \\
\text { (subgrupos). }\end{array}$ \\
\hline & $\begin{array}{l}\text { Enseñanza } \\
\text { Modular }\end{array}$ & $\begin{array}{l}\text { Posibilidad de híbridos en la enseñanza de la Educación Física; sus } \\
\text { características principales: integra en un mismo estilo de enseñanza } \\
\text { intereses con niveles de ejecución. }\end{array}$ \\
\hline & $\begin{array}{c}\text { Programas } \\
\text { Individuales }\end{array}$ & $\begin{array}{l}\text { Son como adaptaciones curriculares por menor o mayor nivel de } \\
\text { ejecución. Todos los alumnos plantean un programa individual. }\end{array}$ \\
\hline & $\begin{array}{l}\text { Enseñanza } \\
\text { programada }\end{array}$ & $\begin{array}{l}\text { Es una enseñanza basada en la que no hay error. Cada uno de los pasos } \\
\text { que tiene que aprender el alumno tiene } 2 \text { características: a su vez es un } \\
\text { programa individual; es una enseñanza en la que no cabe error. El } \\
\text { alumno sólo progresa en función de la superación de las taras.as. }\end{array}$ \\
\hline \multirow{3}{*}{$\begin{array}{l}\text { Enseñanza que } \\
\text { Implica } \\
\text { Cognitivamente } \\
\text { al Alumno }\end{array}$} & $\begin{array}{l}\text { Descubrimiento } \\
\text { Guiado }\end{array}$ & $\begin{array}{l}\text { Este estilo consiste en dar indicios por parte del profesor a los alumnos } \\
\text { para que ellos lleguen a la respuesta deseada. Esto induce al alumno a un } \\
\text { proceso de investigación donde va descubriendo las soluciones. }\end{array}$ \\
\hline & & $\begin{array}{l}\text { Este estilo pretende que el alumno encuentre por si solo la respuesta a } \\
\text { un problema. }\end{array}$ \\
\hline & $\begin{array}{l}\text { Resolución de } \\
\text { Problemas }\end{array}$ & $\begin{array}{l}\text { Conteras (1998) nos dice. La estrategia consiste fundamentalmente en la } \\
\text { búsqueda de alternativas para la resolución de problemas motrices, } \\
\text { estimulando así la creatividad. }\end{array}$ \\
\hline $\begin{array}{l}\text { Enseñanza que } \\
\text { favorece la } \\
\text { Socialización }\end{array}$ & $\begin{array}{c}\text { Estilo } \\
\text { Socializador }\end{array}$ & $\begin{array}{l}\text { Los estilos socializadores, y más concretamente el juego de roles, no } \\
\text { solamente es utilizada en la Didáctica de Educación Física, sino también } \\
\text { se utiliza frecuentemente en otras áreas que persiguen el aprendizaje de } \\
\text { valores. }\end{array}$ \\
\hline $\begin{array}{l}\text { Enseñanza que } \\
\text { promueve la } \\
\text { Creatividad. }\end{array}$ & Estilo Creativo & $\begin{array}{l}\text { El alumno crea sus propios objetivos, contenidos, el pensamiento es } \\
\text { divergente. Necesitan mucho tiempo para observar mejorías y } \\
\text { resultados". }\end{array}$ \\
\hline
\end{tabular}

Fuente: Adaptado de (Fernández-Rivas y Espada-Mateos, 2016)

La aplicación de cada estilo de aprendizaje con su respectiva técnica en primer lugar depende de la destreza con desempeño que se quiere desarrollar; así, por ejemplo, para desarrollar la estimulación perceptiva motriz; se utiliza con más frecuencia estilos como el descubrimiento guiado y asignación de tareas. Para desarrollar capacidades físicas 
básicas, se utiliza con más frecuencia el mando directo y la asignación de tareas Byra, M., Sanchez, B., \& Wallhead, T. L. (2014) Otros autores mencionan en sus respectivas investigaciones que la utilización constante de estilos más creativos como el descubrimiento guiado, socializador, individual, etc. Llevan al aprendizaje efectivo del estudiante.

\section{Conclusiones}

De las distintas investigaciones realizadas, analizadas y contextualizadas con la realidad de nuestras instituciones educativas y la labor que cumplen los docentes de la Educación Física con respecto a la información sobre Estrategias Didácticas de la Educación Física para Desarrollas la Motivación en Estudiantes de Educación Escolar, se han obtenido las siguientes conclusiones:

La utilización de las estrategias de aprendizaje en Educación Física en el ambiente de clases es de vital importancia para impartir una formación integral en las instituciones educativas.

Las estrategias de aprendizaje permiten al docente ser innovadores, creativos y sobre todo salir del aburrimiento y lo rutinario.

Las estrategias de aprendizaje son macro actividades que el docente pone en práctica para cumplir con los fines, metas y objetivos planteados.

La práctica de la motivación en una clase depende de las actividades que realiza de acuerdo a las estrategias planificadas.

El desarrollo de valores y la autoestima estriba directamente del docente en como es el ambiente de clase, la comunicación y sobre todo la orientación que ha planificado.

En la mayoría de instituciones educativas de la Provincia del Cañar existen docentes que están impartiendo clases de Educación Física que no son profesionales en la rama.

El éxito de la formación del estudiante está en que esa educación sea realista, contextual, tomando en cuenta las necesidades, intereses y potencialidades de cada uno de los estudiantes.

La formación integral depende de la aplicación de la normativa vigente por todos los que estamos implícitos en el mismo.

\section{Referencias Bibliográficas}

Ainscow, M. (2001). Desarrollo de escuelas inclusivas. Ideas, propuestas. Narcea, 2001.

Alarcon, T., y Reyno, M. (2012). Estilos de enseñanza en la educación física. Ciencias de la actividad física y deporte, 15-24.

Arjona Garrido, Á., Checa Olmos, J. C., y Pardo García, R. (2016). Educación física y deporte: ¿Instrumentos de integración de inmigrados? Revista de Ciencias Sociales, 10-21.

Byra, M., Sanchez, B., y Wallhead, T. (2014). Behaviors of students and teachers in the command, practice, and inclusion styles of teaching: Instruction, feedback, and activity level. European Physical Education Review, 20(1), 3-19. doi: 10.1177/ 1356336X13495999

Dweck, C. (2013). Self-theories: Their role in motivation, personality, and development. Psychology press.

Escolano, E., y Gerrero, M. (2012). Uso de la estrategia Cognitiva de Aprendizaje en Educación Infantil. Internatonial Jourdal od Developmental. , 275-284.

Fernández, J. (2004). Recuperando el valor ético y político de la pedagogía: Didáctica de la Educación Física. Una perspectiva crítica y transversal. , 315-330.

Fernández-Rivas, M., y Espada-Mateos, M. E. (2016). Frecuencia de utilización y aceptación de estilos de enseñanza en Educación Física..

Gano-Overway, L., y Guivernau, M. (2014). Caring in the gym: Reflections from middle school physical education teachers. European Physical Education Review, 20(2), 264-281. doi: 10.1177/1356336X14524856

González-Cutre, D. (2017). Estrategias didácticas y motivacionales en las clases de educación física desde la teoría de la autodeterminación. Revista de Educación, Motricidad e Investigación(8), 44. doi: 10.33776/remo.v0i8.3268

Llames, R., y Dantas, A. G. (2017). Entrenamiento psicológico deportivo aplicado a una estudiante de oposición. Revista de Psicologia del Deporte, 26, 93-98.

Martos, y al, E. (2014). El desarrollo de la autonomía y la responsabilidad en educación física: Un estudio de caso colaborativo en secundaria. RETOS. Nuevas Tendencias en Educación Física, Deporte y Recreación(26), 3-8.

Ministerio del deporte. (2010). Ley del Deporte Educación Física y Recreación., 17.

Valle, A. (2012). La investigación pedagógica. Habana: Pueblo y Educaciòn.

Vallerand, R. J., Verner-Filion, J., y Paquet, Y. (2015). Passion for sport and exercise. Routledge Companion to Sport and Exercise Psychology, 160-206. doi: 10.4324/9781315880198.ch43

Recibido: 30 de agosto de 2019

Aceptado: 15 de mayo de 2020 